TRANSACTIONS OF THE

AMERICAN MATHEMATICAL SOCIETY

Volume 357, Number 12, Pages 4805-4812

S 0002-9947(05)03866-3

Article electronically published on July 19, 2005

\title{
A SIMPLE ALGORITHM FOR PRINCIPALIZATION OF MONOMIAL IDEALS
}

\author{
RUSSELL A. GOWARD, JR.
}

\begin{abstract}
In this paper, we give a simple constructive proof of principalization of monomial ideals and the global analog. This also gives an algorithm for principalization.
\end{abstract}

\section{INTRODUCTION}

Let $X$ be a scheme of finite type over a field $k$ of arbitrary characteristic. Suppose that $D_{1}, \ldots, D_{h}$ are effective divisors such that $\sum D_{i}$ has simple normal crossings on $X$. Then, in characteristic zero, a simple case of Hironaka's famous theorem on principalization shows that there exists a sequence of blow-ups at non-singular centers principalizing the ideal $\mathcal{I}_{D_{1}}+\ldots+\mathcal{I}_{D_{h}}$ locally generated by the defining equations of the $D_{i}$. That is, if we denote by $\pi: X^{\prime} \longrightarrow X$ the composite of these blow-ups, then the ideal $\left(\sum \mathcal{I}_{D_{i}}\right) \mathcal{O}_{X^{\prime}}$ is a principal ideal defining a divisor with simple normal crossings.

In this paper, we give a simple constructive proof of this result that is valid in any characteristic. We show furthermore that the non-singular centers can always be chosen to be the intersections of precisely two of the prime divisors in the support of the divisor $D_{1}+\ldots+D_{h}$ (or its pullback). In particular, one can principalize a monomial ideal in the polynomial ring $k\left[x_{1}, \ldots, x_{n}\right]$ by performing "monomial" blow-ups.

A basic idea in resolution of singularities is to define invariants which behave well under blowing up. Then the locus of points, or a subvariety thereof, where the invariant is a maximum, is the center of a blow-up. Ideally, the invariants will decrease after blowing up until finally resolution has occured.

We follow that idea here as well. We define a global invariant $(\sigma(X), \tau(X))$ of a pair of divisors $D_{1}, D_{2}$ such that $D_{1}+D_{2}$ has simple normal crossings on $X$. We then show that after a single blow-up $X_{1} \stackrel{\pi}{\longrightarrow} X$ of a monomial center determined by $(\sigma(X), \tau(X))$, the invariant decreases $(\sigma(X), \tau(X))>\left(\sigma\left(X_{1}\right), \tau\left(X_{1}\right)\right)$ in the lexicographic order. Because $(\sigma, \tau) \in \mathbf{N}^{2} \times \mathbf{N}$, this decreasing cannot continue indefinitely and eventually ends in a local principalization of $\mathcal{I}_{D_{1}}+\mathcal{I}_{D_{2}}$. Because the

Received by the editors November 20, 2002

2000 Mathematics Subject Classification. Primary 13A99, 14E99.

The author thanks Steven Dale Cutkosky for his advice and patience as supervisor for the author's Ph.D. thesis, and Karen Smith for her advice and help with numerous corrections to this paper.

(C)2005 American Mathematical Society Reverts to public domain 28 years from publication 
general case easily reduces to the case of two divisors, this produces a simple algorithm for principalization for any number of divisors $D_{i}$, which can be implemented on a computer.

In the proof, the following points will become clear: 1) the algorithm is constructive and independent of a characteristic of the ground field; 2) the centers of the blow-ups are monomial; and 3) only codimension two blow-ups are necessary for principalization. The latter two points do not appear explicitly in the literature as far as we know.

In section 2, we define the invariant $(\sigma(X), \tau(X))$ and explain the ordering on these pairs. We also give some examples to illustrate how $\sigma(X)$ determines the non-principal locus. It will be clear that $(\sigma(X), \tau(X))$ together with an arbitrary ordering of the prime divisors in the support of $D_{1}+\ldots+D_{h}$ uniquely determine the monomial centers of blowing-up in our algorithm.

In section 3, we prove the main results. In section 4, we present the algorithm in pseudocode. In section 5, we apply the algorithm in an example in dimension three.

For a general introduction to blowing-up we refer to [H1]. For a treatment of algorithms of resolution of singularities, we refer to $\mathrm{BM}$, $[\mathrm{EV}]$ and we refer to $\mathrm{H} 2$ for Hironaka's results on resolution.

\section{Definition OF INVARIANT}

Before we define the invariant used in the algorithm, we give an example to motivate the definition.

Example. Let $k$ be a field and consider the monomial ideal $I=\left(x^{a} y^{b}, x^{c} y^{d}\right)$ of $k[x, y]$. When is $I$ a principal ideal? For $I$ to be principal, we must have one monomial dividing the other. This happens when $a \leq c, b \leq d$ or $a \geq c, b \geq d$. Equivalently, $I$ fails to be principal if and only if $a-c$ and $b-d$ have opposite signs. The same idea permeates our definition of the invariant $\sigma$ in the general case.

Suppose that $X$ is a non-singular algebraic variety over a field $k$. We review some well-known terminology in order to fix notation.

Definition 1. An effective divisor $D$ on $X$ is a formal sum of the form $D=$ $\sum_{i=1}^{n} a_{i} E_{i}$, where the $E_{i}$ are irreducible codimension one subvarieties of $X$. The $E_{i}$ are called prime divisors.

Definition 2. A locally principal ideal sheaf $\mathcal{I} \subset \mathcal{O}_{X}$ has simple normal crossings at $p \in X$ if there exist regular parameters $\left(x_{1}, \ldots, x_{r}\right)$ at $p$ such that $\mathcal{I}_{p}=$ $x_{1}^{a_{1}} \cdots x_{r}^{a_{n}} \mathcal{O}_{X, p}$ for some natural numbers $a_{i}$.

An effective divisor $D=\sum_{i=1}^{k} a_{i} E_{i}$ on $X$ has simple normal crossings if its ideal sheaf $\mathcal{I}_{D}=\mathcal{I}_{E_{1}}^{a_{1}} \cdots \mathcal{I}_{E_{k}}^{a_{k}}$ has simple normal crossings at every point $p \in X$.

Definition 3. Let $D_{1}=\sum_{i=1}^{k} a_{i} E_{i}$ and $D_{2}=\sum_{i=1}^{k} b_{i} E_{i}$ be divisors with simple normal crossings on $X$. Then, for each pair of irreducible prime components with non-trivial intersection, define $\sigma\left(E_{i} \cap E_{j}\right)$ by

$$
\sigma\left(E_{i} \cap E_{j}\right)= \begin{cases}\max \left\{\left(\left|a_{i}-b_{i}\right|,\left|a_{j}-b_{j}\right|\right),\left(\left|a_{j}-b_{j}\right|,\left|a_{i}-b_{i}\right|\right)\right\} & \text { if } a_{i}-b_{i} \text { and } a_{j}-b_{j} \\ & \text { have oppposite signs } \\ (-\infty,-\infty) & \text { otherwise }\end{cases}
$$


The max is taken according to the lexicographic order on $\mathbf{N} \times \mathbf{N}$. For example, $(1,3)<(2,5)$ and $(3,1)<(3,2)$. Of course $(-\infty,-\infty)<(i, j)$ for all $i, j \in \mathbf{Z}$.

Definition 4. With the notation given in Definition 3, define

$$
\begin{aligned}
& \sigma(X)=\max \left\{\sigma\left(E_{i} \cap E_{j}\right) \mid E_{i} \cap E_{j} \neq \emptyset, i \neq j\right\}, \\
& \tau(X)=\#\left\{C \subset X \mid C=E_{i} \cap E_{j}, \sigma(C)=\sigma(X)\right\} .
\end{aligned}
$$

It is easy to see that the non-principal locus of $\mathcal{I}_{D_{1}}+\mathcal{I}_{D_{2}}$ is determined by $\sigma$ : it is exactly the union of the $E_{i} \cap E_{j}$ such that $\sigma\left(E_{i} \cap E_{j}\right) \neq(-\infty,-\infty)$ (see Proposition 1). Our algorithm calls for us to blow-up the centers $E_{i} \cap E_{j}$ such that $\sigma\left(E_{i} \cap E_{j}\right)$ is maximal. If $\tau>1$, then there are several possible choices of centers to blow-up. We make this choice unique, for example, by always choosing $E_{a} \cap E_{b}$ so that $(a, b)$ is maximal over all such $(i, j)$ in the lex order on $\mathbf{N} \times \mathbf{N}$.

Example 1. Let $X=\operatorname{Spec}\left(k\left[x_{1}, x_{2}\right]\right)$ and consider the effective divisors $D_{1}=$ $3 E_{1}+4 E_{2}$ and $D_{2}=E_{1}+5 E_{2}$, where $E_{i}=V\left(x_{i}\right)$. Then $\sigma(X)=\sigma\left(E_{1} \cap E_{2}\right)=(2,1)$ and $\tau=1$. We note that the order of the divisors $E_{i}$ does not effect the value $\sigma$. If we change the order, then we still get that $\sigma(X)=(2,1)$.

Note that at the origin $p=E_{1} \cap E_{2}, \mathcal{I}_{D_{1}, p}+\mathcal{I}_{D_{2}, p}=\left(x_{1}^{3} x_{2}^{4}, x_{1} x_{2}^{5}\right)$ is not principal.

Example 2. Let $X=\operatorname{Spec}\left(k\left[x_{1}, x_{2}, x_{3}\right]\right)$. Let $D_{1}=5 E_{1}+3 E_{2}+2 E_{3}$ and $D_{2}=$ $E_{1}+4 E_{2}+E_{3}$, where $E_{i}$ is the divisor whose local equation is $x_{i}=0$. Since $a_{1}-b_{1}$ and $a_{3}-b_{3}$ have the same sign, $\sigma\left(E_{1} \cap E_{3}\right)=(-\infty,-\infty)$. On the other hand, $a_{1}-b_{1}$ and $a_{2}-b_{2}$ have opposite signs, so $\sigma\left(E_{1} \cap E_{2}\right)=(4,1)$. Also $a_{2}-b_{2}$ and $a_{3}-b_{3}$ have opposite signs and $\sigma\left(E_{2} \cap E_{3}\right)=(1,1)$. Then $\sigma(X)=$ $\max \{(1,1),(4,1),(-\infty,-\infty)\}=(4,1)$ and $\tau(X)=1$.

In this case, our algorithm calls for blowing-up the line $E_{1} \cap E_{2}$. Doing so, we introduce a new (exceptional) divisor $E_{4}$ and our divisors become

$$
\begin{aligned}
& \pi_{1}^{*} D_{1}=5 E_{1}+3 E_{2}+2 E_{3}+8 E_{4}, \\
& \pi_{1}^{*} D_{2}=E_{1}+4 E_{2}+E_{3}+5 E_{4} .
\end{aligned}
$$

We abuse notation and write $E_{i}$ for their proper transforms. Then $\sigma\left(E_{2} \cap E_{3}\right)=$ $(1,1), \sigma\left(E_{2} \cap E_{4}\right)=(3,2)$, and all the other pairs of intersecting divisors have $\sigma\left(E_{i} \cap E_{j}\right)=(-\infty,-\infty)$. Hence our next center of blowing-up will be $E_{2} \cap E_{4}$.

\section{Proof of PRINCIPALIZATION}

We begin with the case of two divisors.

Proposition 1. Let $X$ be a non-singular algebraic variety, and let $D_{1}, D_{2}$ be effective divisors on $X$ such that $D_{1}+D_{2}$ has simple normal crossings. Then $\mathcal{I}_{D_{1}}+\mathcal{I}_{D_{2}}$ is principal at $p \in X$ if and only if $\sigma\left(E_{i} \cap E_{j}\right)=(-\infty,-\infty)$ whenever $p \in E_{i} \cap E_{j}$. In particular, $\mathcal{I}_{D_{1}}+\mathcal{I}_{D_{2}}$ is locally principal if and only if $\sigma(X)=(-\infty,-\infty)$.

Proof. Let $x_{1}, \ldots, x_{n} \in \mathcal{O}_{X, p}$ be regular parameters at $p$. Let

$$
\begin{aligned}
& f_{1}=\delta_{1} x_{1}^{a_{1}} \ldots x_{n}^{a_{r}}, \\
& f_{2}=\delta_{2} x_{1}^{b_{1}} \ldots x_{n}^{b_{r}}
\end{aligned}
$$

be local equations for $D_{1}$ and $D_{2}$ with $\delta_{i} \in \mathcal{O}_{X, p}$ units. Suppose $\mathcal{I}_{D_{1}}+\mathcal{I}_{D_{2}}$ is not principal at $p$. Then $\left(f_{1}, f_{2}\right)$ is not principal and it follows that, for some $i, j$ with $i \neq j, a_{i}-b_{i}$ and $a_{j}-b_{j}$ have opposite signs. Otherwise, the $a_{i}-b_{i}$ all are $\leq 0$ or $\geq 0$ which implies $f_{1} \mid f_{2}$ or $f_{2} \mid f_{1}$. Let $E_{i}$ be the component with local equation 
$x_{i}=0$, and let $E_{j}$ be the component with local equation $x_{j}=0$. Then $p \in E_{i} \cap E_{j}$ and $\sigma\left(E_{i} \cap E_{j}\right)>0$. Conversely, suppose $p \in E_{i} \cap E_{j}$ and $\sigma\left(E_{i} \cap E_{j}\right)>0$. Let $x_{i}=0$ and $x_{j}=0$ be local equations for $E_{i}$ and $E_{j}$ in a neighborhood of $p$. Then by definition of $\sigma, a_{i}-b_{i}$ and $a_{j}-b_{j}$ have opposite signs. Hence it follows that $\left(f_{1}, f_{2}\right)$ is not principal, and so $\mathcal{I}_{D_{1}}+\mathcal{I}_{D_{2}}$ is not principal at $p$.

Remark. It follows from the proof of Proposition 1 that if $\mathcal{I}_{D_{1}}+\mathcal{I}_{D_{2}}$ is principal at $p$, then either $\left(\mathcal{I}_{D_{1}}+\mathcal{I}_{D_{2}}\right)_{p}=\left(\mathcal{I}_{D_{1}}\right)_{p}$ or $\left(\mathcal{I}_{D_{1}}+\mathcal{I}_{D_{2}}\right)_{p}=\left(\mathcal{I}_{D_{2}}\right)_{p}$. We will make use of this fact later in the proof of Lemma 1.

Theorem 1. Let $X$ be a non-singular algebraic variety. Suppose $D_{1}$ and $D_{2}$ are effective divisors on $X$ such that $D_{1}+D_{2}$ has simple normal crossings. Suppose that $C \subset X$ is the intersection of two components of $D_{1}+D_{2}$ with $\sigma(C)=\sigma(X)>$ $(-\infty,-\infty)$. Let $\pi: X_{1} \rightarrow X$ be the blow-up of $X$ centered at $C$. Then we have $(\sigma(X), \tau(X))>\left(\sigma\left(X_{1}\right), \tau\left(X_{1}\right)\right)$ in the lexicographic order.

Proof. Write $D_{1}=\sum_{i=1}^{k} a_{i} E_{i}$ and $D_{2}=\sum_{i=1}^{k} b_{i} E_{i}$, where the $E_{i}$ are irreducible components. By relabeling the $E_{i}$ if necessary we may assume that $C=E_{1} \cap E_{2}$. Likewise we may also assume that $a_{1}-b_{1}<0$ and $a_{2}-b_{2}>0$.

Let $\pi: X^{\prime} \longrightarrow X$ be the blow-up of $X$ with center $C$, and let $E$ be the exceptional divisor of $\pi$. Denote by $E_{i}^{\prime}$ the proper transform of $E_{i}$. A simple local computation shows that

$$
\pi^{*} E_{i}= \begin{cases}E_{i}^{\prime}+E & \text { if } i=1,2 \\ E_{i}^{\prime} & \text { if } i>2\end{cases}
$$

Hence

$$
\begin{gathered}
\pi^{*} D_{1}=\left(a_{1}+a_{2}\right) E+\sum_{i=1}^{k} a_{i} E_{i}^{\prime}, \\
\pi^{*} D_{2}=\left(b_{1}+b_{2}\right) E+\sum_{i=1}^{k} b_{i} E_{i}^{\prime} .
\end{gathered}
$$

Now we compute $\sigma$ to show that it has has not increased under this blow-up. For the divisors $E_{i}^{\prime}$ which are transforms of divisors on $X$, we have $\sigma\left(E_{i}^{\prime} \cap E_{j}^{\prime}\right)=$ $\sigma\left(E_{i} \cap E_{j}\right)$ whenever $E_{i}^{\prime}$ and $E_{j}^{\prime}$ intersect. So it suffices to consider pairs where one of the divisors is exceptional. Denote $C_{j}=E \cap E_{j}^{\prime}$. Because the argument is symmetric for $j=1$ or 2 , and likewise for any $j \geq 3$, there are only really two cases to consider.

Case 1. $j=1$. By our initial assumption, $a_{1}-b_{1}<0$. If $a_{1}+a_{2}-\left(b_{1}+b_{2}\right) \leq 0$, then $\sigma\left(C_{1}\right)=(-\infty,-\infty)$ and we are done. So suppose $a_{1}+a_{2}-\left(b_{1}+b_{2}\right)>0$. Then $b_{1}-a_{1}<a_{2}-b_{2}$ and we have

$$
\begin{aligned}
\sigma & =\left(a_{2}-b_{2}, b_{1}-a_{1}\right) \text { and } \\
\sigma\left(C_{1}\right) & =\max \left\{\left(b_{1}-a_{1}, a_{1}+a_{2}-\left(b_{1}+b_{2}\right)\right),\left(a_{1}+a_{2}-\left(b_{1}+b_{2}\right), b_{1}-a_{1}\right)\right\} .
\end{aligned}
$$

Now since $a_{1}-b_{1}$ is negative, we see that

$$
a_{1}+a_{2}-\left(b_{1}+b_{2}\right)<a_{2}-b_{2} .
$$

Since also

$$
a_{2}-b_{2}>a_{1}-b_{1}
$$

we see directly that in either case $\sigma\left(C_{1}\right)$ is strictly less than $\sigma$. 
Case 2. $j \geq 3$. Assume that $a_{3}-b_{3}<0$. (The case when $a_{3}-b_{3}>0$ has a similar argument.) If $a_{1}+a_{2}-\left(b_{1}+b_{2}\right) \leq 0$, then $\sigma\left(E \cap E_{3}\right)=(-\infty,-\infty)$ and we are done. So assume that $a_{1}+a_{2}-\left(b_{1}+b_{2}\right)>0$. As before this implies that $\sigma=\left(a_{2}-b_{2}, b_{1}-a_{1}\right)$. Then there are two possibilities for $\sigma\left(C_{3}\right)=\sigma\left(E \cap E_{3}\right)$. One possibility is that $\sigma\left(C_{3}\right)=\left(a_{1}+a_{2}-\left(b_{1}+b_{2}\right), b_{3}-a_{3}\right)$. In this case, we have $a_{2}-b_{2}>\left(a_{2}-b_{2}\right)+\left(a_{1}-b_{1}\right)$ since $a_{1}-b_{1}$ is negative. Hence $\sigma=\left(a_{2}-b_{2}, b_{1}-a_{1}\right)>$ $\left(a_{1}+a_{2}-\left(b_{1}+b_{2}\right), b_{3}-a_{3}\right)=\sigma\left(C_{3}\right)$ and we are done in this case. The other possibility is that $\sigma\left(C_{3}\right)=\left(b_{3}-a_{3}, a_{1}+a_{2}-\left(b_{1}+b_{2}\right)\right)$. Note that we can assume that $E_{2} \cap E_{3} \neq \emptyset$, otherwise $E_{3}$ does not intersect $E$ at all. Thus by maximality of $\sigma$, $a_{2}-b_{2} \geq b_{3}-a_{3}$. It follows that $\sigma>\sigma\left(C_{3}\right)$ except possibly when $a_{2}-b_{2}=b_{3}-a_{3}$. However this cannot happen since this equality and the fact that $\sigma \geq \sigma\left(E_{2} \cap E_{3}\right)$ would imply $b_{1}-a_{1} \geq b_{3}-a_{3}=a_{2}-b_{2}$, which contradicts the computation above that $a_{2}-b_{2}>b_{1}-a_{1}$.

Now we have shown that the invariant $\sigma$ definitely decreases locally under blowing-up of our chosen center. Thus the global invariant $(\sigma, \tau)$ decreases as well, in the lexicographic order: under the blow-up $\pi: X^{\prime} \longrightarrow X$, either $\sigma\left(X^{\prime}\right)<\sigma(X)$ or $\sigma\left(X^{\prime}\right)=\sigma(X)$ and $\tau\left(X^{\prime}\right)=\tau(X)-1$.

It is now easy to prove the theorem that was mentioned in the Introduction. First, we need the following lemma.

Lemma 1. If $D_{1}, \ldots, D_{h}$ are divisors on a non-singular variety $X$ such that $D_{1}+$ $\cdots+D_{h}$ has simple normal crossings, then $\mathcal{I}_{D_{1}}+\cdots+\mathcal{I}_{D_{h}}$ is locally principal if $\mathcal{I}_{D_{i}}+\mathcal{I}_{D_{j}}$ is locally principal for all pairs $i, j$.

Proof. If $\mathcal{I}_{D_{1}}+\mathcal{I}_{D_{2}}$ is principal at $p$, then either $\left(\mathcal{I}_{D_{1}}+\mathcal{I}_{D_{2}}\right)_{p}=\left(\mathcal{I}_{D_{1}}\right)_{p}$ or $\left(\mathcal{I}_{D_{1}}+\mathcal{I}_{D_{2}}\right)_{p}=\left(\mathcal{I}_{D_{2}}\right)_{p}$, but then either

$$
\left(\mathcal{I}_{D_{1}}+\cdots+\mathcal{I}_{D_{h}}\right)_{p}=\left(\mathcal{I}_{D_{1}}+\mathcal{I}_{D_{3}}+\cdots+\mathcal{I}_{D_{h}}\right)_{p}
$$

or

$$
\left(\mathcal{I}_{D_{1}}+\cdots+\mathcal{I}_{D_{h}}\right)_{p}=\left(\mathcal{I}_{D_{2}}+\mathcal{I}_{D_{3}}+\cdots+\mathcal{I}_{D_{h}}\right)_{p}
$$

and we are done by induction on $h$.

Theorem 2. Let $D_{1}, \ldots, D_{h}$ be effective divisors, such that $\sum D_{i}$ has simple normal crossings. Then there exists a sequence of blow-ups of non-singular centers $\pi: X^{\prime} \rightarrow X$ such that $\left(\mathcal{I}_{D_{1}}+\cdots+\mathcal{I}_{D_{h}}\right) \mathcal{O}_{X^{\prime}}$ is locally principal and defines a simple normal crossing divisor.

Proof. By Lemma 1, we reduce immediately to the case when $h=2$. Fix an ordering of $E_{1}, \ldots, E_{k}$ of the divisors in the support of $D_{1}+D_{2}$. Compute $(\sigma, \tau)$. If $\sigma=(-\infty,-\infty)$, then the ideal $\mathcal{I}_{D_{1}}+\mathcal{I}_{D_{2}}$ is locally principal by Proposition 3.1 and we are done. Otherwise consider all the non-empty intersections $E_{i} \cap E_{j}$ where $\sigma\left(E_{i} \cap E_{j}\right)$ is maximal. From among these, let $C=E_{a} \cap E_{b}$, where $(a, b)$ is maximal in the lexicographic ordering of $\mathbf{N} \times \mathbf{N}$. Let $\pi: X_{1} \longrightarrow X$ be the blow-up of $X$ with center $C$. On $X_{1}$, we have the divisors $E_{1}, \ldots, E_{k}, E_{k+1}$ still with normal crossings, where $E_{k+1}$ is the exceptional divisor of $\pi$ and by abuse of notation we have written $E_{1}, \ldots, E_{k}$ for their proper transforms. The divisors $\pi^{*} D_{1}, \pi^{*} D_{2}$ are made up of the prime divisors $E_{1}, \ldots, E_{k}, E_{k+1}$. By Theorem 1, we have $(\sigma(X), \tau(X))>\left(\sigma\left(X_{1}\right), \tau\left(X_{1}\right)\right)$. We can then repeat this process on $X_{1}$, using the divisors $\pi^{*} D_{1}, \pi^{*} D_{2}$. Since $(\sigma, \tau) \in \mathbf{N}^{2} \times \mathbf{N}$, this process must eventually stop. 
When it stops, we have $(\sigma, \tau)=(-\infty,-\infty)$, and $\mathcal{I}_{D_{1}}+\mathcal{I}_{D_{2}}$ has been principalized. The proof is complete.

Corollary 1. Let $R=k\left[x_{1}, \ldots, x_{n}\right]$ be a polynomial ring over a field $k$. If $I \subset R$ is a monomial ideal, then there exists a monomial ideal $J \subset R$ such that $X=$ Proj $R[J t]$ is non-singular and $I \mathcal{O}_{X}$ is locally principal.

Proof. Let $X=\operatorname{Spec} R$. Suppose $I=\left(m_{1}, \ldots, m_{j}\right)$ with each $m_{t}$ a monomial in the $x_{i}$. Then each monomial defines an effective divisor and their sum has normal crossing support on $X$. By the above theorem, there exists a sequence of blow-ups

$$
X_{l} \stackrel{\pi_{l}}{\longrightarrow} X_{l-1} \stackrel{\pi_{l-1}}{\longrightarrow} \ldots \longrightarrow X_{1} \stackrel{\pi_{1}}{\longrightarrow} X=\mathbf{A}_{k}^{n}
$$

so that $I \mathcal{O}_{X_{l}}$ is principal and defines a simple normal crossings divisor. Let $X_{l}=X$ and $\pi=\pi_{l} \circ \pi_{l-1} \circ \ldots \circ \pi_{1}$ and define $J=I \mathcal{O}_{X}$. Then $J$ is the desired monomial ideal.

In another paper $[\mathbf{G}$, we show, given the monomial ideal $I \subset R$, how to explicitly construct a monomial ideal $J \subset I$ such that $X=\operatorname{Proj} R[J t]$ is non-singular and $I \mathcal{O}_{X}$ is locally principal.

\section{Algorithm: Principalization of monomial ideals}

INPUT: $(X, \mathcal{E}, \mathcal{D})$, where $X$ is a non-singular algebraic variety, $\mathcal{E}=\left\{E_{i}\right\}$ is a collection of prime divisors in simple normal crossings, and $\mathcal{D}=\left\{D_{1}, \ldots, D_{h}\right\}$ is a finite collection of effective divisors supported on $\mathcal{E}$.

OUTPUT: a sequence of blow-ups

$$
X_{l} \stackrel{\pi_{l}}{\longrightarrow} X_{l-1} \stackrel{\pi_{l-1}}{\longrightarrow} \ldots \longrightarrow X_{1} \stackrel{\pi_{1}}{\longrightarrow} X_{0}=X
$$

of non-singular centers such that $\mathcal{I O}_{X_{l}}$ is principal where $\mathcal{I}=\mathcal{I}_{D_{1}}+\cdots+\mathcal{I}_{D_{h}}$. Furthermore, each center will be of the form $E \cap F$ where $E$ and $F$ are either the proper transforms of the $E_{i}$ or exceptional divisors (or their proper transforms) of the maps.

INITIALIZATION: $X_{p}:=X_{0}:=X, \mathcal{E}:=\left\{E_{1}, \ldots, E_{n}\right\}, \mathcal{D}=\left\{D_{1}, \ldots, D_{h}\right\}$.

STEP 1: For $(a, b) \in\{1, \ldots, h\} \times\{1, \ldots, h\}, a \neq b$ DO.

STEP 2: Consider divisors $D_{a}=\sum a_{i} E_{i}$ and $D_{b}=\sum b_{i} E_{i}$. If $E_{i} \cap E_{j} \neq \emptyset$, then compute

$$
\sigma\left(E_{i} \cap E_{j}\right)= \begin{cases}\max \left\{\left(\left|a_{i}-b_{i}\right|,\left|a_{j}-b_{j}\right|\right),\left(\left|a_{j}-b_{j}\right|,\left|a_{i}-b_{i}\right|\right)\right\} & \text { if } a_{i}-b_{i} \text { and } a_{j}-b_{j} \\ & \text { have oppposite signs } \\ (-\infty,-\infty) & \text { otherwise. }\end{cases}
$$

Here the maximum is taken with respect to the lex order on $\mathbf{N} \times \mathbf{N}$. Compute $\sigma\left(X_{p}\right)=\max \sigma\left(E_{i} \cap E_{j}\right)$. Set $\tau\left(X_{p}\right)=\# E_{i} \cap E_{j}$ such that $\sigma\left(E_{i} \cap E_{j}\right)=\sigma\left(X_{p}\right)$.

WHILE $\sigma\left(X_{p}\right)>(-\infty,-\infty)$ DO

Set $C=E_{c} \cap E_{d}$ where $(c, d)$ is uniquely determined as the max in the lex order among the indices $i, j$ such that $\sigma\left(E_{i} \cap E_{j}\right)=\sigma\left(X_{p}\right)$. Let $\pi_{p+1}: X_{p+1} \longrightarrow X_{p}$ be the blow-up of $X_{p}$ with center $C$. Let $E_{n+1}=\pi_{p+1}^{-1}(C)$ be the exceptional divisor of this map.

For each $E_{i} \in \mathcal{E}$, set $E_{i}:=$ the proper transform of $E_{i}$ on $X_{p+1}$. For each $D_{i} \in \mathcal{D}$, set $D_{i}:=\pi_{p+1}^{*} D_{i}$. Set $\mathcal{E}:=\mathcal{E} \cup\left\{E_{n+1}\right\}, n:=n+1$, and $p:=p+1$. 


\section{GO TO STEP 2}

OTHERWISE: $\sigma\left(X_{p}\right)=(-\infty,-\infty)$ and composite map $X_{p} \longrightarrow$ $X_{0}$ is a sequence of blow-ups which principalizes $\mathcal{I}_{D_{a}}+\mathcal{I}_{D_{b}}$.

\section{GO TO STEP 1}

\section{EXAMPLE IN DIMENSION 3}

Example. Let $X=\mathbf{A}_{k}^{3}, k$ an arbitrary field. Let $E_{1}, E_{2}$ and $E_{3}$ be any prime divisors in $\mathbf{A}_{k}^{3}$ intersecting in simple normal crossings - for example, the coordinate planes. Let $D_{1}=E_{1}+3 E_{2}+2 E_{3}$ and $D_{2}=2 E_{1}+E_{2}+E_{3}$. Then

$$
\begin{aligned}
\sigma\left(E_{1} \cap E_{2}\right) & =(2,1), \\
\sigma\left(E_{1} \cap E_{3}\right) & =(1,1), \\
\sigma\left(E_{2} \cap E_{3}\right) & =(-\infty,-\infty),
\end{aligned}
$$

and $\sigma(X)=(2,1), \tau(X)=1$ so $C_{1}=E_{1} \cap E_{2}$ will be the center of the first blow-up.

Let $\pi_{1}: X_{1} \longrightarrow X_{0}=X$ be the blow-up of $X$ with center $C_{1}$ and let $E_{-1}=$ $\pi_{1}^{-1}\left(C_{1}\right)$ be the exceptional divisor. Then

$$
\begin{aligned}
& \pi_{1}^{*} D_{1}=4 E_{-1}+E_{1}^{\prime}+3 E_{2}^{\prime}+2 E_{3}^{\prime}, \\
& \pi_{1}^{*} D_{2}=3 E_{-1}+2 E_{1}^{\prime}+E_{2}^{\prime}+E_{3}^{\prime} .
\end{aligned}
$$

Note that $E_{1}^{\prime}$ and $E_{2}^{\prime}$ do not intersect at all. We compute $\sigma\left(E_{-1} \cap E_{1}^{\prime}\right)=(1,1)$, $\sigma\left(E_{1}^{\prime} \cap E_{3}^{\prime}\right)=(1,1)$, and all other pairs have $\sigma=(-\infty,-\infty)$. Hence $\left(\sigma\left(X_{1}\right), \tau\left(X_{1}\right)\right)$ $=((1,1), 2)$. To decide what center to blow-up, we use the lex order on the indices $(i, j)$ to choose the center $C_{2}=E_{1}^{\prime} \cap E_{3}^{\prime}$.

Let $\pi_{2}: X_{2} \longrightarrow X_{1}$ be the blow-up of $X_{1}$ with center $C_{2}=E_{1}^{\prime} \cap E_{3}^{\prime}$. Let $E_{-2}=\pi_{2}^{-1}\left(C_{2}\right)$ be the exceptional divisor. Then

$$
\begin{aligned}
& \pi_{2}^{*} D_{1}=3 E_{-2}+4 E_{-1}^{\prime}+E_{1}^{\prime \prime}+3 E_{2}^{\prime \prime}+2 E_{3}^{\prime \prime}, \\
& \pi_{2}^{*} D_{2}=3 E_{-2}+3 E_{-1}^{\prime}+2 E_{1}^{\prime \prime}+E_{2}^{\prime \prime}+E_{3}^{\prime \prime} .
\end{aligned}
$$

Note that among the intersecting pairs of divisors, we have for all such pairs $\sigma=$ $(-\infty,-\infty)$ except for $\sigma\left(E_{-1}^{\prime} \cap E_{1}^{\prime \prime}\right)=(1,1)$. Hence $\sigma\left(X_{2}\right)=(1,1)$ and $\tau\left(X_{2}\right)=1$. Again we see that

$$
\begin{aligned}
\left(\sigma\left(X_{2}\right), \tau\left(X_{2}\right)\right) & =((1,1), 1) \\
& <((1,1), 2) \\
& =\left(\sigma\left(X_{1}\right), \tau\left(X_{1}\right)\right) .
\end{aligned}
$$

Our next center is thus $C_{3}=E_{-1}^{\prime} \cap E_{1}^{\prime \prime}$.

Let $\pi_{3}: X_{3} \longrightarrow X_{2}$ be the blow-up of $X_{2}$ with center $C_{3}=E_{-1}^{\prime} \cap E_{1}^{\prime \prime}$. Let $E_{-3}=\pi_{3}^{-1}\left(C_{3}\right)$ be the exceptional divisor. Then

$$
\begin{aligned}
& \pi_{2}^{*} D_{1}=5 E_{-3}+3 E_{-2}^{\prime}+4 E_{-1}^{\prime \prime}+E_{1}^{\prime \prime \prime}+3 E_{2}^{\prime \prime \prime}+2 E_{3}^{\prime \prime \prime}, \\
& \pi_{2}^{*} D_{2}=5 E_{-3}+3 E_{-2}^{\prime}+3 E_{-1}^{\prime \prime}+2 E_{1}^{\prime \prime \prime}+E_{2}^{\prime \prime \prime}+E_{3}^{\prime \prime \prime} .
\end{aligned}
$$

One now easily verifies that $\sigma=(-\infty,-\infty)$ for these two divisors because whenever two of the prime divisors in their support intersect, the differences of the corresponding coefficients have the same sign. In other words, the ideal $\mathcal{I}_{D_{1}}+\mathcal{I}_{D_{2}}$ is locally principal. Note however that this ideal is not globally principal. 


\section{REFERENCES}

[BM] Bierstone, E., Milman, P., Canonical Desingularization in Characteristic Zero by BlowingUp the Maximal Strata of a Local Invariant, Ivent. Math., 128 (1997), 207-302. MR1440306 (98e:14010)

[EV] Encinas, S., Villamayor, O., A Course on Constructive Desingularization and Equivariance, Resolution of Singularities: A Research Textbook, Birkhauser, Progress in Mathematics, v. 181 (2000). MR.1748620 (2001g:14018)

[G] Goward, Russell, A., A Principalizing Ideal of a Monomial Ideal, preprint.

[H1] Hartshorne, R., Algebraic Geometry, Graduate Texts in Mathematics, 52, Springer-Verlag, 1977. MR0463157 (57:3116)

[H2] Hironaka, H., Resolution of Singularities of an Algebraic Variety Over a Field of Characteristic Zero, Annals of Math., Vol. 79, 1964. MR0199184 (33:7333)

Department of Mathematics, University of Michigan-Ann Arbor, Ann Arbor, MichiGAN 48109-1109 